\title{
A Case of Primary Sjögren's Syndrome Presenting as Mass-Like Encephalitis, With Progression to Neuromyelitis Optica Spectrum Disorder
}

\author{
Hyun-Jung LEE, ${ }^{1}$ Sung Hae CHANG, ${ }^{2}$ Eun Ha KANG,${ }^{3}$ Yun Jong LEE, ${ }^{3}$ Yeong Wook SONG, ${ }^{4}$ You-Jung HA ${ }^{3}$ \\ ${ }^{1}$ Department of Internal Medicine, Seoul National University Hospital, Seoul, South Korea \\ ${ }^{2}$ Department of Internal Medicine, Division of Rheumatology, Soonchunhyang University Cheonan Hospital, Cheonan, South Korea \\ ${ }^{3}$ Department of Internal Medicine, Division of Rheumatology, Seoul National University Bundang Hospital, Seongnam, South Korea \\ ${ }^{4}$ Department of Internal Medicine, Division of Rheumatology, Seoul National University Hospital, Seoul, South Korea
}

\begin{abstract}
Neuromyelitis optica is an idiopathic inflammatory demyelinating disease of the central nervous system (CNS) that predominantly affects the optic nerves and spinal cord. With the discovery of the pathogenic anti-aquaporin-4 (AQP4) antibody, the disease was recognized as part of a spectrum of autoimmune diseases that target AQP4, collectively referred to as neuromyelitis optica spectrum disorder (NMOSD). NMOSD consists of conditions that affect various parts of the CNS with the AQP4 antibody. In this article, we report a 43-year-old female patient who was initially diagnosed with primary Sjögren's syndrome (pSS) with CNS involvement, but was later diagnosed with overlapping pSS and NMOSD, which required more intensive treatment. The patient presented with fever, headache, dysarthria, and left-side weakness, and brain imaging showed a mass-like edematous lesion in the right frontoparietal region. She also complained of xerostomia and was diagnosed with pSS by salivary scintigraphy, anti-Sjögren's syndrome A positivity, and minor salivary gland biopsy. Under the diagnosis of pSS with CNS involvement in the form of tumefactive encephalitis, she was treated with high-dose steroids and monthly intravenous cyclophosphamide therapy. However, three months later, she developed a sudden decrease in right visual acuity and had right optic neuritis. Her serum was positive for the anti-AQP4 antibody, and she was finally diagnosed with overlapping NMOSD and pSS. She was treated with steroid pulse therapy and plasmapheresis. Therefore, in patients with pSS presenting with cerebral white matter lesions, even when optic neuritis or myelitis is absent, evaluations for the anti-AQP4 antibody should be considered to detect and treat NMOSD accordingly.

Keywords: Neuromyelitis optica spectrum disorder; Sjögren's syndrome; tumefactive encephalitis.
\end{abstract}

Primary Sjögren's syndrome (pSS) is a systemic autoimmune disease characterized by chronic inflammation of exocrine glands. At least onethird of pSS patients present with extraglandular systemic features including neurologic, articular, pulmonary, or renal manifestations. ${ }^{1,2}$ The prevalence of neurologic manifestations in pSS is reported to range from 2 to $60 \% .^{3}$ Sensory polyneuropathies are the most common neurologic manifestations, while central nervous system (CNS) involvement in SS is less common. ${ }^{4}$ In rare cases, neurologic manifestations of pSS can present as acute meningoencephalitis.,
Neuromyelitis optica spectrum disorder (NMOSD) is a chronic autoimmune demyelinating disease of the CNS, usually of the optic nerve and spinal cord, that is associated with serum anti-aquaporin-4 (AQP4) immunoglobulin G antibodies, which have a sensitivity of $60 \sim 70 \%$ and specificity of $99 \%$ for the disease. ${ }^{6}$ Recent studies have reported the overlap of NMOSD and other autoimmune diseases including pSS and systemic lupus erythematosus, suggesting that these diseases are related..$^{7-10}$

In this article, we describe a rare case of a patient with pSS and NMOSD, who presented as

Received: September 02, 2016 Accepted: November 29, 2016 Published online: April 04, 2017

Correspondence: You-Jung Ha, MD. Department of Internal Medicine, Division of Rheumatology, Seoul National University Hospital, 13620 Seoul, South Korea.

Tel: +82-31-787-7081 e-mail: haayou@hanmail.net

@2017 Turkish League Against Rheumatism. All rights reserved. 


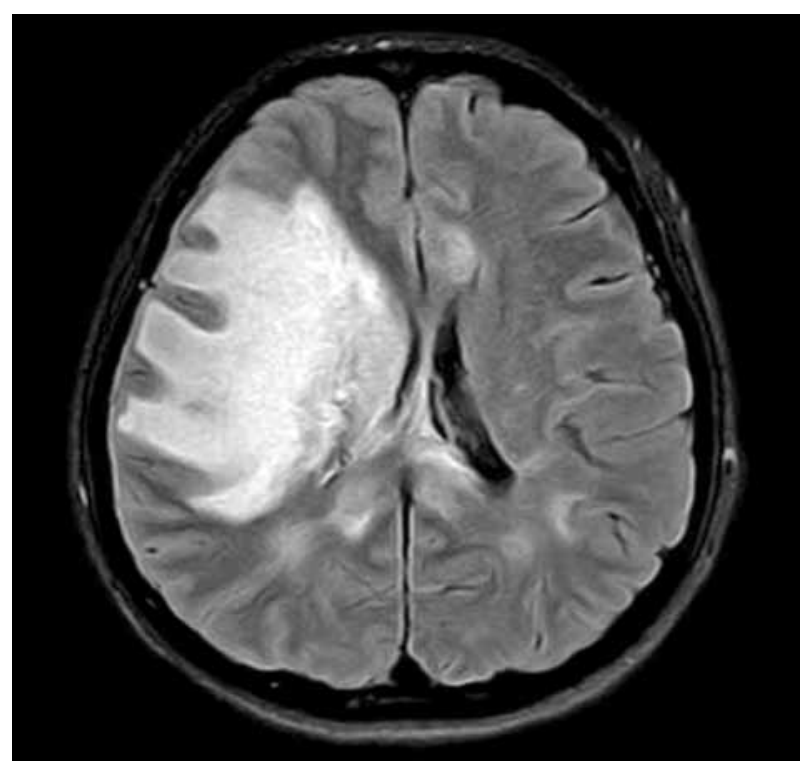

Figure 1. Brain magnetic resonance imaging revealed that lesion was largely edema with high $\mathrm{T}_{2}$ signal intensity and no primary mass, accompanied by multiple small lesions in corpus callosum and periventricular white matter.

acute mass-like encephalitis and later developed optic neuritis.

\section{CASE REPORT}

A 43-year-old female patient presented with acute-onset fever, headache, dysarthria, amnesia, left-side weakness, and dysesthesia. Initial brain computed tomography scans showed a $7 \mathrm{~cm}$, mass-like lesion in the right frontoparietal region, suggesting a brain tumor. However, brain magnetic resonance imaging (MRI) revealed that the lesion was largely edema with high $\mathrm{T}_{2}$ signal intensity and no primary mass, accompanied by multiple small lesions in the corpus callosum and periventricular white matter (Figure 1). The cerebrospinal fluid profile showed the following: white blood count, $16 / \mathrm{mm}^{3}$ and protein, $55 \mathrm{mg} / \mathrm{dL}$. Considering the possibility of encephalitis, empirical antibiotics and acyclovir were administrated, but no improvements in her symptoms or MRI were observed. Under suspicion of tumefactive multiple sclerosis and other demyelinating diseases, intravenous steroid pulse (methylprednisolone $1 \mathrm{~g}$ ) therapy for five days and subsequent high-dose prednisolone was administered. The patient recovered enough to walk and only a tingling discomfort remained in the left hand. She was referred to a rheumatologist to screen for underlying autoimmune diseases. She had complained of dry mouth for 10 years, but denied having dry eyes. Blood tests showed the following: positive for antinuclear antibody 1:320 (speckled pattern) and anti-SSA/Ro, and negative for anti-SSB/La, anti-double-stranded deoxyribonucleic acid, rheumatoid factor, and anti-phospholipid antibodies, with normal C3/C4 levels. Salivary scintigraphy showed decreased uptake in the submandibular and parotid glands (Figure 2a), and examinations for dry eyes were positive. A minor salivary gland biopsy showed lymphoplasmacytic infiltration with a focus score $>2$ (Figure 2b). Therefore, she was diagnosed as having pSS with CNS involvement in the form of tumefactive encephalitis. Monthly intravenous cyclophosphamide therapy was started and glucocorticoids were tapered slowly.
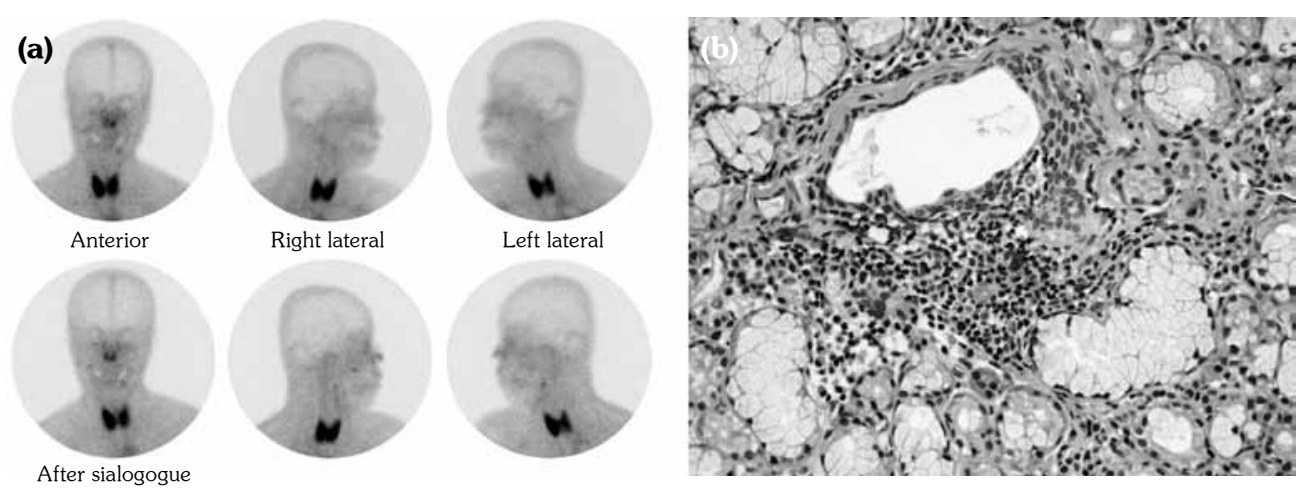

Figure 2. (a) Salivary scintigraphy showed decreased uptake in the submandibular and parotid glands. (b) A minor salivary gland biopsy showed lymphoplasmacytic infiltration with a focus score $>2$ (H-Ex400). 
A written informed consent was obtained from the patient.

Two weeks after the third cycle of intravenous cyclophosphamide and a reduction of prednisolone to $20 \mathrm{mg}$, the patient visited the emergency room with a severe headache. However, brain MRI showed no new lesions, only chronicstage encephalitis with atrophic changes in the previously observed multiple edematous lesions. Two days later, she complained of right-eye pain with a sudden decrease in right visual acuity. Ophthalmological examinations showed a right relative afferent pupillary defect, decreased right visual field, and mild swelling of the right optic disc. Orbital MRI showed interval development of right optic neuritis (Figure 3a, b). Whole-spine MRI showed no evidence of myelitis in the spinal cord. Steroid pulse therapy was administered for three days and plasmapheresis was performed five times. Her vision improved enough for her to read large letters. Results of tests for the antiAQP4 antibody were positive, and the patient was diagnosed with NMOSD related to pSS. Follow-up orbital MRI after four months showed decreased right optic nerve swelling with disappeared contrast-enhancement, suggesting chronic stage of optic neuritis (Figure 3c, d).

\section{DISCUSSION}

Our patient presented with fever, neurological symptoms, and a mass-like edematous lesion in the frontoparietal region suggestive of encephalitis, thus NMOSD was not initially suspected. She was diagnosed with pSS with CNS involvement. However, she went on to develop optic neuritis, and tests for AQP4-immunoglobulin G antibodies were positive, leading to the diagnosis of NMOSD.

According to the 2006 diagnostic criteria for NMOSD, bilateral optic neuritis or longitudinally extensive transverse myelitis lesions were characteristic findings, and normal brain MRI or only nonspecific white matter lesions was a key supportive criterion. ${ }^{11}$ However, brain involvement in NMOSD is increasingly being recognized, ${ }^{12,13}$ and up to $60 \%$ of patients with NMOSD had brain abnormalities on follow-up. ${ }^{6}$ Typical patterns of brain involvement in NMOSD, although not pathognomonic, include lesions of
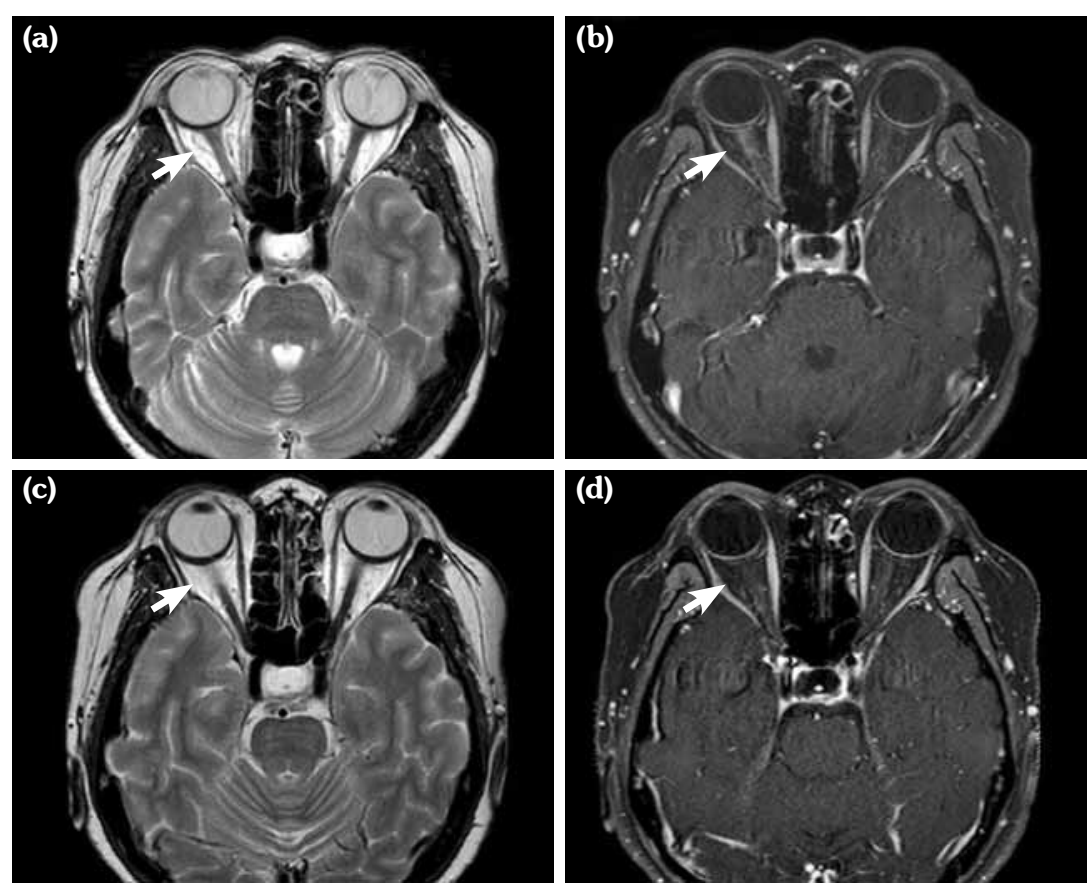

Figure 3. (a, b) Orbital magnetic resonance imaging showed interval development of right optic neuritis. (c, d) Follow-up orbital magnetic resonance imaging after four months showed decreased right optic nerve swelling with disappeared contrast-enhancement, suggesting chronic stage of optic neuritis. 
the dorsal medulla/area postrema, periependymal regions in the brainstem, diencephalic structures, or cerebral hemispheres or long lesions spanning much of the corpus callosum or corticospinal tracts, corresponding to regions of high AQP4 expression. Large, confluent, or tumefactive cerebral lesions may suggest NMOSD, but in isolation may not be discernible from atypical multiple sclerosis lesions. ${ }^{6}$

The coexistence of NMOSD and pSS is increasingly being reported, and most cases present with optic neuritis or transverse myelitis, making them more easily recognizable. ${ }^{7,10,14,15}$ Cases of pSS with NMOSD that present with symptomatic cerebral white matter lesions, without optic neuritis or myelitis, are rare and hard to diagnose. In addition, although the anti-SSA/Ro antibody is frequently detected in patients with NMOSD, one series reported that only $2 \%$ of patients with NMOSD met the criteria for pSS.9,16 To our knowledge, two cases of pSS and NMOSD that were positive for AQP4 antibodies with only cerebral white matter lesions have been reported to date, and one was proven by brain biopsy to be more compatible with NMOSD than with pSS. ${ }^{14,17}$ Our patient satisfied the 2012 classification criteria for $\mathrm{pSS},{ }^{18}$ and her minor salivary gland biopsy showed typical histopathologic findings of pSS.

Therefore, physicians should consider NMOSD in patients with pSS who present with cerebral white matter lesions and show characteristic MRI findings of NMOSD, even when optic neuritis or spinal cord lesions are not detected. As in this case, such brain lesions can be the initial manifestation of combined pSS and NMOSD, thus checking for the antiAQP4 antibody may be helpful for establishing a differential diagnosis and the proper treatment strategy.

\section{Declaration of conflicting interests}

The authors declared no conflicts of interest with respect to the authorship and/or publication of this article.

\section{Funding}

The authors received no financial support for the research and/or authorship of this article.

\section{REFERENCES}

1. Rehman HU. Sjögren's syndrome. Yonsei Med J 2003;44:947-54.

2. Vitali C, Bombardieri S, Jonsson R, Moutsopoulos HM, Alexander EL, Carsons SE, et al. Classification criteria for Sjögren's syndrome: a revised version of the European criteria proposed by the AmericanEuropean Consensus Group. Ann Rheum Dis 2002;61:554-8.

3. García-Carrasco M, Ramos-Casals M, Rosas J, Pallarés L, Calvo-Alen J, Cervera R, et al. Primary Sjögren syndrome: clinical and immunologic disease patterns in a cohort of 400 patients. Medicine (Baltimore) 2002;81:270-80.

4. Chai J, Logigian EL. Neurological manifestations of primary Sjogren's syndrome. Curr Opin Neurol 2010;23:509-13.

5. Matsui Y Takenouchi T, Narabayashi A, Ohara K, Nakahara T, Takahashi T. Childhood Sjögren syndrome presenting as acute brainstem encephalitis. Brain Dev 2016;38:158-62.

6. Wingerchuk DM, Banwell B, Bennett JL, Cabre P, Carroll W, Chitnis $\mathrm{T}$, et al. International consensus diagnostic criteria for neuromyelitis optica spectrum disorders. Neurology 2015;85:177-89.

7. Kahlenberg JM. Neuromyelitis optica spectrum disorder as an initial presentation of primary Sjögren's syndrome. Semin Arthritis Rheum 2011;40:343-8.

8. Wingerchuk DM, Weinshenker BG. The emerging relationship between neuromyelitis optica and systemic rheumatologic autoimmune disease. Mult Scler 2012;18:5-10.

9. Park JH, Hwang J, Min JH, Kim BJ, Kang ES, Lee KH. Presence of anti-Ro/SSA antibody may be associated with anti-aquaporin-4 antibody positivity in neuromyelitis optica spectrum disorder. J Neurol Sci 2015;348:132-5.

10. Qiao L, Wang Q, Fei Y, Zhang W, Xu Y, Zhang Y, et al. The Clinical Characteristics of Primary Sjogren's Syndrome With Neuromyelitis Optica Spectrum Disorder in China: A STROBE-Compliant Article. Medicine (Baltimore) 2015;94:1145.

11. Wingerchuk DM, Lennon VA, Pittock SJ, Lucchinetti CF, Weinshenker BG. Revised diagnostic criteria for neuromyelitis optica. Neurology 2006;66:1485-9.

12. Pittock SJ, Lennon VA, Krecke K, Wingerchuk DM, Lucchinetti CF, Weinshenker BG. Brain abnormalities in neuromyelitis optica. Arch Neurol 2006;63:390-6.

13. Chan KH, Tse CT, Chung CP, Lee RL, Kwan JS, Ho $\mathrm{PW}$, et al. Brain involvement in neuromyelitis optica spectrum disorders. Arch Neurol 2011;68:1432-9.

14. Min JH, Kim HJ, Kim BJ, Lee KW, Sunwoo IN, Kim SM, et al. Brain abnormalities in Sjogren syndrome with recurrent CNS manifestations: association with neuromyelitis optica. Mult Scler 2009;15:1069-76. 
15. Jayarangaiah A, Sehgal R, Epperla N. Sjögren's syndrome and neuromyelitis optica spectrum disorders (NMOSD)--a case report and review of literature. BMC Neurol 2014;14:200.

16. Pittock SJ, Lennon VA, de Seze J, Vermersch P, Homburger HA, Wingerchuk DM, et al. Neuromyelitis optica and non organ-specific autoimmunity. Arch Neurol 2008;65:78-83.

17. Sawada J, Orimoto R, Misu T, Katayama T, Aizawa $\mathrm{H}$, Asanome A, et al. A case of pathology-proven neuromyelitis optica spectrum disorder with Sjögren syndrome manifesting aphasia and apraxia due to a localized cerebral white matter lesion. Mult Scler 2014;20:1413-6.

18. Shiboski SC, Shiboski CH, Criswell L, Baer A, Challacombe S, Lanfranchi $\mathrm{H}$, et al. American College of Rheumatology classification criteria for Sjögren's syndrome: a data-driven, expert consensus approach in the Sjögren's International Collaborative Clinical Alliance cohort. Arthritis Care Res (Hoboken) 2012;64:475-87. 